\title{
IDENTIFICAÇÃO DO PERFIL FÍSICO-FUNCIONAL DE IDOSOS DE UMA INSTITUIÇÃO DE LONGA PERMANÊNCIA
}

\author{
Vania Fernanda Clemente Agner Fisioterapeuta, mestranda no \\ Programa de Ciências da Saúde na \\ Universidade Federal de São Paulo.
}

Anna Raquel Silveira Gomes

Luana Pereira Paz

Fisioterapeuta, pós-graduada na

Residência Multiprofissional em

Atenção Hospitalar - Urgência e

Emergência do Hospital de Clínicas

da Universidade Federal do Paraná.

\section{Clynton Lourenço Correa}

Fisioterapeuta, professor adjunto do curso de Fisioterapia da Universidade Federal do Rio de Janeiro. Diretor científico da Associação Brasileira de Fisioterapia Neurofuncional.

Endereço para correspondência: clyntoncorrea@gmail.com

\begin{abstract}
Resumo
Objetivo: Identificar o perfil físico-funcional de idosos de uma Instituição de Longa Permanência para Idosos (ILPI) e apresentá-lo a seus gestores. Metodologia: análise dos registros de saúde de 42 idosos (> 60 anos), coletando-se: idade; sexo; tempo de institucionalização; situação de renda; escolaridade; medicamentos; tabaco; uso de fralda geriátrica; dispositivo para locomoção; serviço de fisioterapia; histórico de quedas e doenças. Foram realizadas 31 avaliações, utilizando Mini-Exame do Estado Mental (MEEM) para estado cognitivo; Questionário de Avaliação Funcional Multidimensional de Idoso; Índice de Massa Corporal; Escala de Equilíbrio de Berg (EEB); goniometria de membros inferiores para amplitude de movimento (ADM) e o teste de sentar e levantar em 30 segundos para força muscular de membros inferiores (FMMI). Foram analisadas médias, desvio padrão e porcentagens. Resultados: idosos com 75 \pm 10 anos; $74 \%$ mulheres; tempo de institucionalização $20 \pm 16$ anos e baixa escolaridade. Todos usavam medicações, 59\% utilizavam cadeira de rodas e $67 \%$ realizaram fisioterapia anteriormente. Foram encontradas prevalências de: tabagismo (19\%); hipertensão arterial (65\%) e diabetes mellitus (26\%). Encontraramse mais idosos utilizando fralda geriátrica (50\%) do que diagnosticados com incontinência urinária (18\%). O escore da EEB foi de 12 \pm 16 , representando alto risco de quedas, porém, em $69 \%$ dos prontuários não constavam informações sobre quedas. Foi detectada diminuição da FMMI (5 \pm 4 repetições) e na ADM para todos os movimentos analisados. Conclusão: o grupo avaliado necessita de intervenções multiprofissionais, pois apresenta risco à complicações secundárias evitáveis. Os dados deste estudo, apresentados para equipe gestora da ILPI, contribuíram para a contratação de 01 fisioterapeuta para essa instituição.
\end{abstract}

Palavras-chaves: Idosos fragilizados; Instituição de longa permanência para idoso; Avaliação geriátrica; Fisioterapia (especialidades). 


\title{
IDENTIFICATION OF PHYSICAL AND FUNCTIONAL PROFILE OF INSTITUTIONALIZED ELDERLY FROM A NURSING HOME
}

\begin{abstract}
Objective: Identify physical and functional profile of elderly from a nursing home $(\mathrm{NH})$ and present to their managers. Methodology: It was analyzed health records of 42 elderly (>60 years) to collect the following data: age; gender; institutionalization's time; income status; education level; medications; tobacco; use of geriatric diaper; device for locomotion; physical therapy support; history of falls and diseases. It was done 31 assessments, applying: Mini-Mental State Examination (MMSE) to identify cognition status; Older Americans Resources and Services; Body Mass Index; Berg Balance Scale (BBS); lower limb's range of motion (ROM) (goniometer) and 30s chairstand test (CT) to evaluate lower limb strength. Descriptive statistics (means, standard deviations and percentages) were used. Results: Elderly were $75 \pm 10$ years; $74 \%$ of women; institutionalization's time was $20 \pm 16$ years and low level of education. All elderly were using medications and 59\% using wheelchair and $67 \%$ had participated from physiotherapy sessions. It was found smoking prevalence (19\%); systemic arterial hypertension (65\%) and diabetes mellitus (26\%). More elderly were using geriatric diaper (50\%) than urinary incontinence diagnosed (18\%). EEB showed score of $12 \pm 16$, representing high risk of falls; however, the health records (69\%) did not indicate fall's information. Muscle strength (CT, $5 \pm 4$ repetitions) and ROM was decreased in lower limbs. Conclusion: the elderly assessed needs specialized multi interventions, because presented risk of secondary complications avoidable. The outcomes presented for the employers contributed to the admission of 01 physiotherapist for this $\mathrm{NH}$.
\end{abstract}

Keywords: Frail elderly; Geriatric long-term care institution; Geriatric assessment; Physicaltherapy (specialty).

\section{INTRODUÇÃO}

Existem atualmente no Brasil 19,7 milhões de idosos, representando 10,7\% da população, sendo que as estimativas apontam que em 2050 a população brasileira será composta por 19\% de idosos. ${ }^{(1)}$ Como consequência do aumento da população idosa, ocorre também o acréscimo do número de idosos institucionalizados, bem como aumento na ocorrência de morbidades e incapacidades. $^{(2,3)}$ Estudos sugerem que entre 1985 e 2060 o número de idosos institucionalizados no Brasil aumentará de 1,3 para 4,5 milhões. ${ }^{(2)}$ O número total de idosos residentes em Instituição de Longa Permanência para Idosos (ILPI) no Brasil é desconhecido, porém, no Paraná este número chega a 6,5 mil. $^{(4)}$

O desempenho físico-funcional é relacionado, dentre outros fatores, ao estado cognitivo, equilíbrio, força muscular e mobilidade. ${ }^{(5)} \mathrm{O}$ declínio do desempenho físico-funcional em idosos é decorrente de fatores ambientais, alterações fisiológicas decorrentes do processo de envelhecimento, ou ainda do estilo de vida adotado pelos idosos ao longo da vida, bem como, de condições sócio-demográficas, físicas, clínicas e cognitivas do indivíduo. ${ }^{(5)}$

Sendo assim, investigar as condições e as modificações físico-funcionais que acontecem durante o processo de envelhecimento são imprescindíveis, especialmente em idosos 
institucionalizados, os quais apresentam comumente déficits funcionais mais expressivos quando comparados aos não institucionalizados. ${ }^{(6)}$ Por exemplo, dificuldades físico-funcionais, perda de autonomia, do suporte familiar, da identidade na comunidade e contexto psicossocial desfavorável podem causar dificuldades nas atividades de vida diária e nas habilidades cognitivas, aumentar o risco de quedas, gerar complicações nas condições de saúde e mais gastos com serviços de saúde, que podem ser evitáveis. ${ }^{(6)}$ Em revisão sistemática recente, que reportou os aspectos fortemente relacionados com a institucionalização, encontrou-se: idade avançada $(\mathrm{OR}=1,31$ até 8,34$)$; não morar em sua própria casa $(\mathrm{OR}=2,61)$; baixa percepção subjetiva do estado geral de saúde $(\mathrm{OR}=1,48$ até 1,67); limitações funcionais $(\mathrm{OR}=1,3$ até 1,78); limitações cognitivas $(\mathrm{OR}=1,44$ até 1,50$)$; diagnóstico de demência $(\mathrm{OR}=16,7){ }^{(7)}$

Nesse contexto, justifica-se essa pesquisa, pois os cuidados fora do núcleo familiar fica sob a responsabilidade de ILPI, que deve assegurar o bem estar que inclui as condições físicofuncionais e ambientais. Assim, o presente estudo teve como objetivo identificar o perfil físicofuncional de idosos residentes de uma ILPI na cidade de Paranaguá, Paraná - Brasil, e apresentar os resultados a seus gestores, visando melhorias nas condições de assistência à saúde desses idosos.

\section{MÉTODOS}

Estudo analítico observacional transversal, aprovado pelo Comitê de Ética em Pesquisa do Setor de Ciências da Saúde da Universidade Federal do Paraná - CAAE 0028.0.091.000-09, seguiu as Normas Éticas previstas na Resolução n¹96, de 10/10/1996, do Conselho Nacional de Saúde.

No período de pesquisa, novembro de 2009, a ILPI contava com 45 pessoas institucionalizadas, sendo 42 idosos. O quadro de funcionários contratados para prestarem serviços diariamente, totalizavam 13, sendo: 2 técnicos de enfermagem, 1 secretário e 10 cuidadores, os quais eram responsáveis pela assistência no cuidado diário, higienização e alimentação dos idosos. Dentre esses, apenas 2 tinham capacitação para cuidados com idosos. A instituição também contava com 1 médico e 1 fisioterapeuta, os quais realizavam voluntariamente atendimentos uma vez por semana. A ILPI onde a pesquisa foi realizada, conta com recursos financeiros de filantropia e das Secretarias Municipal e Estadual de Saúde e do 
Ministério da Saúde. Os idosos que residiam nessa instituição, os quais eram beneficiados com algum recurso financeiro, contribuíam com $70 \%$ de sua renda.

Para participar da pesquisa, os idosos deveriam contemplar os seguintes critérios de inclusão: idade igual ou superior a 60 anos, ambos os gêneros, residir nessa instituição, preservação da comunicação verbal, aceitação e concordância em participar do estudo, por meio da assinatura do Termo de Consentimento Livre e Esclarecido (TCLE). Foram excluídos da pesquisa indivíduos que não atendessem aos critérios de inclusão supracitados e que estivessem ausentes do local durante o período de coleta de dados - por motivo de visita à casa de familiares ou internações hospitalares, ou os que se recusassem a participar da pesquisa.

O coordenador da pesquisa realizou capacitação técnica prévia aos avaliadores, antes da coleta de dados, para padronização dos métodos de pesquisa. Os avaliadores deste estudo foram alunos voluntários, que cursavam graduação em Fisioterapia na Universidade Federal do Paraná. O estudo foi realizado em duas fases, apresentadas no fluxograma do estudo - figura 01.

$\mathrm{Na}$ primeira fase - Caracterização dos Idosos, foram realizadas consultas aos prontuários dos 42 idosos, para coletar informações referentes à idade, sexo, tempo de institucionalização, situação de renda, escolaridade, medicação, uso ou não de: tabaco, fralda geriátrica, dispositivo de locomoção, do serviço de fisioterapia e, também sobre o histórico de quedas e doenças.

A segunda fase - Avaliação Físico-Funcional, com objetivo de verificar a função físicofuncional dos idosos, teve a participação de 31 idosos, os quais aceitaram participar da pesquisa. A sequência de avaliação foi padronizada para todos os idosos, os quais foram avaliados individualmente numa sala reservada. Foram utilizados para todos os idosos os instrumentos de avaliação de pesquisa com testes físicos e escalas funcionais, a saber: Mini Exame do Estado Mental, Questionário de Avaliação Funcional Multidimensional de Idosos, Índice de Massa Corporal, Goniometria Ativa de Membros Inferiores, Teste de Sentar e Levantar em 30segundos e Escala de Equilíbrio de Berg. 


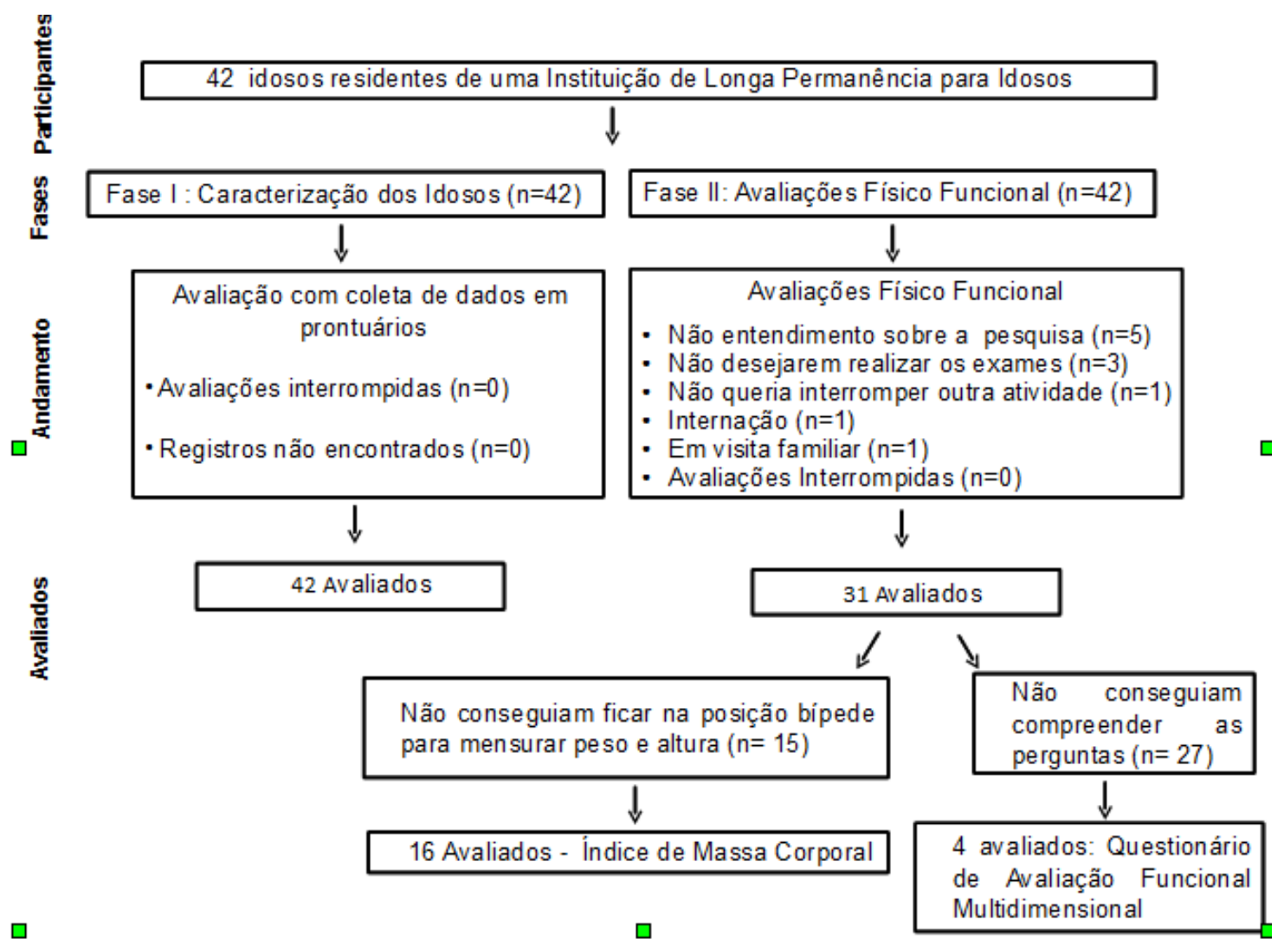

Figura 1 - Fluxograma do Estudo

O primeiro teste aplicado, para verificar a condição cognitiva dos idosos, foi o Mini Exame do Estado Mental (MEEM), o qual consiste em perguntas que determinam a orientação temporal, espacial, memória imediata, cálculo, evocação das palavras, nomeação, repetição, comando verbal, leitura e uso da motricidade fina. Ele apresenta escores diferenciados para indivíduos com diferentes níveis de escolaridade. Os seguintes valores de corte foram aplicados: 20 para analfabetos; 25 para indivíduos com 1 a 4 anos de escolaridade, 26 para 5 a 8 anos, 28 para 9 a 11 anos e 29 para mais de 11 anos de escolaridade. ${ }^{(8)}$

Após a aplicação do MEEM, os idosos que apresentaram o escore de estado mental correspondente com os valores de corte esperado para a idade, responderam o Questionário de Avaliação Funcional Multidimensional de Idosos (QARS), nos tópicos Saúde Física (questões 37-55, 81, 82 e 96 - compreensão do processo saúde-doença) e Atividades de Vida Diária (AVDs), questões 56 a 69 - sobre auto cuidado, higienização e atividades simples do cotidiano), 
com objetivo verificar a condição da saúde física e verificar o nível de atividades de vida diária dos idosos dessa ILPI. ${ }^{(9)}$

Em seguida, foram mensuradas as medidas de peso com balança digital simples, e altura, com o paciente na posição bípede e a mensuração por meio da fita métrica, para posterior cálculo e classificação do Índice de Massa Corporal (IMC), considerando-se a razão massa corporal atual $(\mathrm{kg})$ e o quadrado da estatura $\left(\mathrm{m}^{2}\right){ }^{(10)}$

Os testes físicos e escalas funcionais como a Goniometria Ativa de Membros Inferiores, Teste de Sentar e Levantar em 30 segundos e Escala de Equilíbrio de Berg foram realizados no último momento.

A Goniometria Ativa de Membros Inferiores, com o uso do goniômetro, foi o instrumento utilizado para avaliar a mobilidade das articulações dos membros inferiores. Foram mensurados os seguintes ângulos das articulações dos membros inferiores: quadris - flexão, extensão, adução, abdução, rotação medial e lateral; dos joelhos - flexão, realizada a partir do movimento de extensão de joelho; dos tornozelos - movimentos de flexão dorsal, flexão plantar, abdução e adução. ${ }^{(11)}$

O Teste de Sentar e Levantar em 30 segundos foi utilizado para avaliar a força muscular funcional dos membros inferiores, por meio de repetições do movimento de levantar-se e sentarse de uma cadeira durante um período de 30 segundos. ${ }^{(12)}$

A Escala de Equilíbrio de Berg (EEB) foi empregada para avaliar o equilíbrio e o risco de quedas em idosos. Foram executadas 14 tarefas, as quais foram classificadas de 0 a 4 , sendo que 0 representava a incapacidade e 4 capacidade total para a realização da tarefa. As tarefas são: 1) Transferência da posição sentada para posição em pé; 2) Permanecer em pé sem apoio; 3) Permanecer sentado sem apoio; 4) Transferência da posição em pé para posição sentada; 5) Transferências; 6) Permanecer em pé com os olhos fechados; 7) Permanecer em pé com os pés juntos; 8) Alcançar a frente com os braços estendidos; 9) Pegar um objeto do chão; 10) Virar-se para olhar para trás; 11) Girar 360 graus; 12) Posicionar os pés alternadamente no degrau; 13) Permanecer em pé com um pé à frente; 14) Permanecer em pé sobre um pé. Essas tarefas quantificam a habilidade do indivíduo em fazer transferências, provas estacionárias, alcance funcional, componentes rotacionais e equilíbrio com base de sustentação diminuída. Foi utilizado um cronômetro para marcar o tempo de realização das tarefas dos itens 2, 3, 6, 7, 13 e 14, em segundos. Para avaliação da tarefa 8 utilizou-se uma régua de $30 \mathrm{~cm}$ e foi solicitado ao sujeito 
que fizesse flexão de um dos ombros a $90^{\circ}$, estendesse os dedos da mão e tentasse alcançar a frente, o mais longe possível, sobre a régua mas sem tocá-la. A medida registrada foi à distância que os dedos alcançaram, quando o participante se inclinou para frente, o máximo que ele conseguisse. Foi também utilizada cadeira com braço, caso o sujeito necessitasse o uso do braço para apoiar-se, durante a realização dos itens 1,4 e 5 . Uma caixa de sapato (40 cm de comprimento, $20 \mathrm{~cm}$ de largura e $15 \mathrm{~cm}$ de altura) de papelão vazia foi utilizada como objeto a ser pego no chão, para o item 9. Uma escada com degraus de altura padrão foi usada para o item 12. A EEB tem como escore máximo 56 pontos, sendo que cada ponto a menos é associado a um aumento de 3 a $4 \%$ no risco de quedas, de 54 a 46 pontos a alteração de um ponto é associada ao aumento de 6 a $8 \%$ de risco, sendo que abaixo de 36 pontos o risco de quedas é de $100 \% .^{(13)}$

Os dados obtidos foram avaliados por meio do programa estatístico SAS system e Statistica 6.0 Stat Soft@, e descritos em porcentagem, média e desvio padrão.

Após o término deste estudo, os resultados obtidos foram apresentados à direção da ILPI, para que profissionais e gestores em saúde tivessem mais subsídios para planejar ações em saúde voltadas à essa população.

\section{RESULTADOS}

Quanto aos resultados da primeira fase: Caracterização dos Idosos Institucionalizados, a média de idade foi de 75,3 $\pm 10,5$ anos e o tempo de institucionalização médio dessa população foi de 16,0 $\pm 20,6$ anos. Os resultados da caracterização dos idosos, descritos por meio de consulta em registros de prontuários, estão descritos na Tabela 1. 
Tabela 1 - Caracterização dos idosos institucionalizados, segundo indicadores físico funcionais e socioeconômicos de saúde, de acordo com os prontuários $(n=42)$

\begin{tabular}{|c|c|c|}
\hline Variável & $\begin{array}{l}\text { Frequência } \\
\mathbf{N}\end{array}$ & $\begin{array}{l}\text { Porcentagem } \\
\%\end{array}$ \\
\hline \multicolumn{3}{|l|}{ Sexo } \\
\hline Feminino & 31 & 73,8 \\
\hline Masculino & 11 & 26,2 \\
\hline Total & 42 & 100 \\
\hline \multicolumn{3}{|l|}{ Refere ter Benefício } \\
\hline Sim & 39 & 92,8 \\
\hline Não & 3 & 7,2 \\
\hline Total & 42 & 100 \\
\hline \multicolumn{3}{|l|}{ Escolaridade } \\
\hline Analfabetismo & 15 & 35,4 \\
\hline Ensino Fundamental Incompleto & 23 & 54,8 \\
\hline Ensino Fundamental Completo & 4 & 9,8 \\
\hline Total & 42 & 100 \\
\hline \multicolumn{3}{|l|}{ Tabagismo (atual) } \\
\hline Sim & 8 & 19,0 \\
\hline Não & 34 & 81,0 \\
\hline Total & 42 & 100 \\
\hline \multicolumn{3}{|l|}{ Uso de medicações } \\
\hline Sim & 42 & 100 \\
\hline Não & 0 & 0,0 \\
\hline Total & 42 & 100 \\
\hline \multicolumn{3}{|l|}{ Uso de fralda geriátrica } \\
\hline Sim & 21 & 50 \\
\hline Não & 21 & 50 \\
\hline Total & 42 & 100 \\
\hline \multicolumn{3}{|l|}{ Uso de cadeira de rodas } \\
\hline Sim & 25 & 59,5 \\
\hline Não & 17 & 40,5 \\
\hline Total & 42 & 100 \\
\hline \multicolumn{3}{|c|}{ Utilização do Serviço de Fisioterapia - nos últimos 10 anos } \\
\hline Sim & 28 & 66,6 \\
\hline Não & 17 & 33,4 \\
\hline Total & 42 & 100 \\
\hline
\end{tabular}

O histórico de quedas foi uma informação relevante, pois verifica a condição física e funcional do idoso. $\mathrm{Na}$ análise dos prontuários sobre o histórico de quedas, 69,0\% apresentaram 
ausência dessa informação. Nos 31,0\% (n=13) dos prontuários que continham a informação sobre quedas, 53,8\% dos idosos apresentavam episódio de queda no último ano.

A prevalência de doenças também foi investigada para verificar a situação de saúde física dos idosos, e está descrita na Tabela 2.

Tabela 2 - Prevalência de doenças dos idosos institucionalizados de acordo com os prontuários avaliados $(n=42)$

\begin{tabular}{lc}
\hline Doença & Prevalência (\%) \\
\hline Hipertensão Arterial & 64,71 \\
Diabetes Mellitus & 26,47 \\
Desnutrição & 20,59 \\
Acidente Vascular Encefálico & 20,59 \\
Doenças da visão & 20,59 \\
Distúrbios mentais & 20,59 \\
Doenças reumatológicas & 17,65 \\
Incontinência urinária & 17,65 \\
Doenças aparelho digestório & 14,71 \\
Doenças auditivas & 8,82 \\
Doenças respiratórias & 8,82 \\
Depressão & 5,88 \\
Sequela de poliomielite & 5,88 \\
Distúrbios de linguagem & 5,56 \\
Doenças cardíacas & 2,94 \\
Câncer & 2,94 \\
Alzheimer & 2,94 \\
Parkinson & 2,94 \\
\hline
\end{tabular}

Os resultados da Segunda Fase: Perfil Físico-Funcional dos Idosos Institucionalizados ( $n=31)$, contemplam a descrição dos resultados dos testes de cognição; perfil físico-funcional de 
saúde; índice de massa corporal; força muscular e amplitude de movimento de membros inferiores; equilíbrio e risco de quedas.

Quanto ao Estado Mental desses idosos, 87,0\% apresentaram alterações no comportamento cognitivo, sendo a média do escore dos indivíduos analfabetos de 9,7士8,1, com

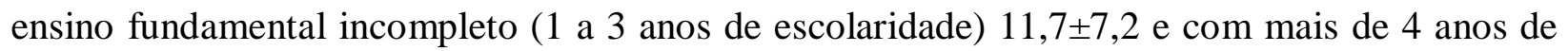
escolaridade $7,3 \pm 4,5$.

Apenas 4 idosos foram avaliados com o questionário OARS, devido ao comprometimento cognitivo dessa amostra. Nesse sentido, os dados coletados são insuficientes para predizer, de acordo com o questionário OARS, o perfil de Saúde Físico-Funcional desse grupo populacional.

Quanto aos valores do IMC, foram avaliados apenas 16 idosos - devido à mensuração da balança na posição bípede. A média desse índice foi de $23,8 \pm 3,5$, sendo que nenhum dos idosos apresentou desnutrição e $31,2 \%$ pode ser considerado com sobrepeso.

Os dados encontrados referentes à amplitude de movimento de articular de membros inferiores estão na Tabela 3, sendo que os resultados para todos os movimentos articulares avaliados, desse grupo de idosos, apresentam valores inferiores aos de referência.

Tabela 3 - Amplitude de movimento articular dos membros inferiores - movimento ativo $\left(^{\circ}\right)$

\begin{tabular}{lccc}
\hline Movimento ativo & $\begin{array}{c}\text { Valores } \\
\text { Referência } *\end{array}$ & $\begin{array}{c}\text { Membro Inferior } \\
\text { Direito } \\
\text { Média } \pm \text { DP }\end{array}$ & $\begin{array}{c}\text { Membro Inferior } \\
\text { Esquerdo } \\
\text { Média } \pm \text { DP }\end{array}$ \\
\hline Flexão quadril & 90 & $53,05 \pm 21,82$ & $44,58 \pm 17,43$ \\
Extensão quadril & 10 & $8,57 \pm 3,51$ & $5,50 \pm 3,39$ \\
Abdução quadril & 45 & $17,42 \pm 8,42$ & $16,17 \pm 9,62$ \\
Adução quadril & 15 & $12,81 \pm 5,47$ & $12,83 \pm 5,95$ \\
Rotação interna quadril & 45 & $19,00 \pm 9,29$ & $16,33 \pm 7,43$ \\
Rotação externa quadril & 45 & $17,00 \pm 9,80$ & $17,03 \pm 7,38$ \\
Flexão joelho & 149 & $94,75 \pm 26,97$ & $79,67 \pm 35,56$ \\
Flexão plantar tornozelo & 45 & $30,15 \pm 9,41$ & $25,07 \pm 11,18$ \\
Flexão dorsal tornozelo & 20 & $13,50 \pm 4,31$ & $11,92 \pm 3,70$ \\
Eversão tornozelo & 20 & $10,89 \pm 3,72$ & $10,32 \pm 4,41$ \\
Inversão tornozelo & 40 & $19,00 \pm 10,75$ & $16,11 \pm 7,94$ \\
\hline
\end{tabular}

*Valores de referência em graus, de acordo com Marques (2003). 
A média de vezes que os idosos conseguiram sentar-se e levantar-se da cadeira, pelo

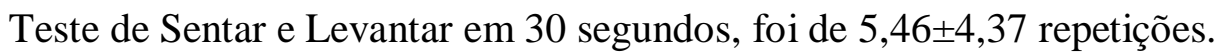

O escore médio encontrado após aplicação da EEB foi $12 \pm 16$, demonstrou que esses idosos apresentaram alto risco de quedas, pois o escore da escala igual ou inferior a 36 prediz um risco de quedas de $100 \% .^{(13)}$

Após a descrição dos resultados da pesquisa, houve uma reunião com os gestores e funcionários da Instituição, com objetivo de apresentar dos resultados encontrados e discutir estratégias de melhorias das condições de saúde dos idosos. Ao final da reunião, sugeriu-se que os resultados da pesquisa fossem apresentados, pelos gestores da instituição - com caráter de corresponsabilização, na Conferência Municipal do Idoso e nos Projetos Sociais, para que se conseguissem recursos financeiros para melhorar a qualidade de vida dos idosos dessa ILPI. Pouco tempo depois da apresentação destes resultados houve a contratação de um fisioterapeuta para a ILPI.

\section{DISCUSSÃO}

Os idosos avaliados tinham média de idade $75 \pm 10$ anos, na sua maioria mulheres $73,8 \%$, taxa mais elevada que num outro estudo, o qual apresentou predomínio de 57,3\% de mulheres entre os residentes de ILPI no Brasil. ${ }^{(14)}$

Apesar do alto índice $(66,6 \%)$, dos residentes, apresentarem registros nos prontuários, quanto à realização de atendimento fisioterapêutico anterior, ainda foi encontrada alta prevalência de risco de quedas, uso de cadeira de rodas para se locomover, diminuição de força muscular e de amplitude de movimentos articulares.

Entende-se que apesar da instituição contar com o serviço voluntário de fisioterapia, esse acontecia com baixa frequência, não contemplando as demandas dos idosos, os quais necessitam não somente de tratamento fisioterapêutico, mas de um programa multiprofissional de prevenção e tratamento de incapacidades. ${ }^{(15)}$

As doenças mais prevalentes nos idosos desta ILPI foram: hipertensão arterial 64,7\% e diabetes mellitus 26,4\%, demonstrando que estão superiores as médias dos estudos sobre prevalência de hipertensão arterial e diabetes mellitus em idosos com mais de 60 anos. ${ }^{(16,17)}$ É importante lembrar que essas doenças são crônicas e podem desencadear complicações 
secundárias e limitantes, como acidente vascular encefálico, infarto, cegueira e perda da função de articulações distais do corpo. ${ }^{(18)}$ Assim, seria indicado para esta ILPI, que fossem seguidas as Diretrizes da Política Nacional de Doenças Crônicas Não-Transmissíveis, as quais recomendam acompanhamento multiprofissional contínuo, com caráter de mudança de estilo de vida e prevenção de complicações secundárias. ${ }^{(18)}$

Percebe-se que esse grupo de idosos, em que 31,2\% apresentaram sobrepeso, necessita de um profissional de nutrição para orientar cuidadores e idosos, diagnosticar e prestar assistência nutricional. $^{(10)}$

A prevalência de tabagistas $(19,05 \%)$ mostrou-se superior ao estudo que apresenta o percentual de fumantes idosos na população brasileira de $9,1 \% .^{(19)}$

Observa-se que há necessidade de intervenções na saúde mental desses idosos, pois a média do escore encontrado por meio da aplicação do Mini-Exame de Estado Mental mostrou que $87,1 \%$ dos indivíduos apresentaram comprometimentos cognitivos. O estado cognitivo dos idosos avaliados encontrou-se bem acima de estudo que reportou 27,5\% de idosos com de sintomas comportamentais, em idosos residentes de ILPI. ${ }^{(20)}$

Sobre o uso de fralda geriátrica, há um conflito de dados, pois 17,6\% dos idosos receberam diagnóstico de incontinência urinária, porém - 50\% dos idosos faziam uso diário de fraldas. Provavelmente, isso acontece na maioria de vezes, porque o número de cuidadores é reduzido, o que faz com que esses cuidadores não tenham tempo para transportar os idosos ao vaso sanitário, contribuindo para a utilização de fraldas geriátricas ${ }^{(21)}$

Os resultados apontaram perda de mobilidade em todas as articulações de membros inferiores avaliadas, corroborando com estudos onde se identificam que envelhecimento acarreta redução da amplitude de movimentos de forma geral, promovendo a exposição epidemiológica para quedas. $^{(22)}$

A média de vezes que os idosos conseguiram sentar-se e levantar-se da cadeira, pelo

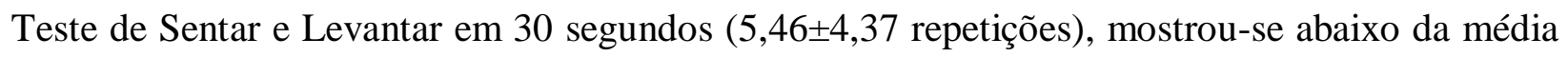
$7,2 \pm 2,6$, avaliada em outro estudo para idosos não praticantes de atividades físicas. ${ }^{(12)}$

A prevalência de quedas nessa população foi de 53,8\%, demonstrando-se maior que em outros estudos de idosos institucionalizados. ${ }^{(23)}$ Os dados mostram alta prevalência de quedas em idosos, sendo mais expressiva em idosos institucionalizados, e revelam a necessidade de prevenção de fatores de risco modificáveis. ${ }^{(23)}$ 
Estudos sugerem que as quedas podem desencadear fraturas, o que diminui a capacidade funcional de idosos e precisam ser prevenidas. ${ }^{(24)}$ Sabe-se que há eficácia do treino de potência, uma intervenção que pode ser realizada pelo fisioterapeuta, para melhorar força e potência muscular, flexibilidade, equilíbrio e capacidade funcional, promovendo redução do risco de quedas em idosos. ${ }^{(22,25)}$

Os resultados do presente estudo demonstram que a maioria dos avaliados, são idosas, de baixa escolaridade e apresentaram alta prevalência de doenças crônicas, risco nutricional, alto índice de tabagismo, comprometimento cognitivo, uso inadequado de fralda geriátrica, mobilidade articular e força muscular reduzidas, alto risco de quedas e falta de assistência fisioterapêutica.

Os dados encontrados nessa pesquisa foram apresentados, pelos pesquisadores, à direção da ILPI e após essa apresentação, os gestores da instituição procuraram projetos sociais a fim de conseguir recursos financeiros para as contratações e capacitações de profissionais, para a melhoria do perfil físico-funcional e cognitivo dos idosos institucionalizados. A ampliação do quadro de funcionários aconteceu com recursos do Projeto Rotary Club.

No mês de janeiro de 2012 o quadro de funcionários da ILPI passou a ser constituído por 15 funcionários contratados: 3 técnicos de enfermagem, 1 secretário, 1 fisioterapeuta e 10 cuidadores. Mais 3 cuidadores realizaram capacitação técnica específica. A ILPI ainda contava, nesse período, com 1 voluntário médico, que continua realizando atendimento uma vez por semana. Observa-se que os dados dessa pesquisa proporcionaram aumento no quadro de funcionários dessa ILPI, mas ainda há necessidade de incremento de recursos humanos e de capacitação desses profissionais.

Este estudo tem limitações, já que o tamanho amostral foi reduzido e a amostra foi escolhida por conveniência, o que reduz o poder de inferência e generalização dos desfechos encontrados. Sugere-se para futuros estudos, avaliações em mais de uma ILPI, escolhidas de forma aleatória bem como a utilização de instrumentos de avaliação padrão ouro, tais como dinamômetro isocinético; ressonância nuclear magnética; baropodometria, plataforma de força, de maneira a investigar a força e massa muscular, variáveis relacionadas ao equilíbrio e risco de quedas. São necessários outros estudos para avaliar se houve melhora nos indicadores de saúde dessa amostra, após o incremento do número de funcionários. 


\section{CONCLUSÃO}

O estudo identificou o perfil físico-funcional dos idosos institucionalizados de uma ILPI em Paranaguá-PR. O grupo avaliado necessita de intervenções multiprofissionais, pois apresenta risco à complicações secundárias evitáveis. Esse estudo contribuiu para o aumento do número de funcionários, incluindo um fisioterapeuta nesta ILPI. No entanto, ainda há necessidade de incremento do número de profissionais e da realização de capacitação para os cuidadores dos idosos de maneira a melhorar o perfil físico-funcional dos idosos.

\section{REFERÊNCIAS}

1. Carvalho JAM, Rodrígues-Wong LL. A transição da estrutura etária da população brasileira na primeira metade do século XXI. Cad. saúde pública. 2008;24(3):597-605.

2. Veras R. Fórum Envelhecimento populacional e as informações de saúde do PNAD: demandas e desafios contemporâneos. Introdução. Cad. saúde pública, 2007;23(10):2463-66.

3. Vitorino LM, Paskulin LMG, Viana LAC. Qualidade de vida de idosos em instituição de longa permanência. Rev. Latino-Am. Enfermagem. 2012;20(6):1186-95.

4. IPARDES - Instituto Paranaense de Desenvolvimento Econômico e Social. Instituições de longa permanência para idosos: caracterização e condições de atendimento. Curitiba; 2008.

5. Van der Velde N, Stricker BH, Pols HA, Van der Cammen TJ. Risk of falls after withdrawal of fall-risk-increasing drugs: a prospective cohort study. Br J Clin Pharmacol. 2007;63(2):232-7.

6. Weening-Dijksterhuis E, Greef MH, Scherder EJ, Slaets JP, Van Der Schans CP. Frail institutionalized older persons: a comprehensive review on physical exercise, physical fitness, activities of daily living, and quality-of-life. Am J Phys Med Rehabil. 2011;90(2):156-68.

7. Luppa M, Luck T, Weyerer S, König H, Brähler E, Riedel-heller SG. Prediction of institutionalization in the elderly. A systematic review. Age ageing. 2010;39:31-38.

8. Bruki SMD, Nitrini R, Caramelli P, Bercolucci HF, Okamo IH. Sugestões para o uso do MINI-EXAME do estado mental no Brasil. Arq. Neuropsiquiaria. 2003;61(3-B):777-781.

9. Blay SL, Ramos LR, Mari J. Validity of a Brazilian version of the Older Americans Resources and Services (OARS) mental health screening questionnaire. J. am. geriatr. soc.1988;36(8):68792. 
10. Santos DM, Sichieri R. Índice de massa corporal e indicadores antropométricos de adiposidade em idosos. Rev. saúde pública. 2005;39(2):163-168.

11. Marques AM. Manual de goniometria. 2a ed. Barueri, SP: Manole; 2003.

12. Smith WN, Del Rossi G, Adams JB, Abderlarahman KZ, Asfour SA, Roos BA et al. Simple equations to predict concentric lower-body muscle power in older adults using the 30-second chair-rise test: a pilot study. Clin Interv Aging. 2010; 9(5)173-80.

13. Miyamoto ST, Lombardi Junior I, Berg KO, Ramos LR, Natour J. Brazilian version of the Berg Balance Scale, 2004. Braz. j. med. biol. res. 2004;37:1411-1421.

14. Camarano AA, Kanso S. As instituições de longa permanência para idosos no Brasil. Rev. bras. estud. popul. 2010;27(1):233-235.

15. Montenegro SMRS, Silva CAB. Os Efeitos de um programa de fisioterapia como promotor de saúde na capacidade funcional de mulheres idosas institucionalizadas. Rev. bras. geriatr. gerontol. 2007,10(2):161-178.

16. Souza LJ, Chalita FEB, Reis AF, Teixeira CL, Neto CG, Bastos FJTDS et al. Prevalência de diabetes mellitus e fatores de risco em Campos dos Goytacazes, RJ. Arq bras. endocrinol. metab. 2003;47(1):69-74.

17. Passos VMA, Assis TD, Barreto SM. Hipertensão arterial no Brasil: estimativa de prevalência a partir de estudos de base populacional. Epidemiol. serv. saúde. 2006; 15(1):35-45.

18. Malta DC, Cezário AC, Moura L, Morais Neto OL, Silva Junior JB. A construção da vigilância e prevenção das doenças crônicas não transmissíveis no contexto do Sistema Único de Saúde. Epidemiol. serv. saúde. 2006;15(1):47- 65.

19. Malta DC, Moura EC, Silva AS, Oliveira PPV, Silva, LCS. Prevalência do tabagismo em adultos residentes nas capitais dos estados e no Distrito Federal, Brasil, 2008. J bras. pneumol. 2010;36(1):75-83.

20. Onder G, Carpenter I, Finne-soveri H, Gindin J, Frijters D, Henrard JC et al. Assessment of nursing home residents in Europe: the Services and Health for Elderly in Long TERm care (SHELTER) study. BMC health serv. res. 2012;12(5):1-10.

21. Moreira MD, Caldas CP. A importância do cuidador no contexto da saúde do idoso. Esc. Anna Nery Rev. Enferm 2007;11(3):520-525.

22. Gallon D, Rodacki ALF, Hernandez SG, Drabovski B, Outi T, Bittencourt LR et al. The effects of stretching on the flexibility, muscle performance and functionality of institutionalized older women. Braz. j. med. biol. res. 2011;44(3):229-235. 
23. Siqueira FV, Facchini LA, Silveira DS, Piccini RX, Tomasi E, Thumé E et al. Prevalence of falls in elderly in Brazil: a country wide analysis. Cad. saúde pública. 2011;27(9):1819-1826.

24. Piirtola M, Löppönen M, Vahlberg T, Isoaho R, Kivelä SL, Räihä I. Fractures as an Independent Predictor of Functional Decline in Older People: A Population-Based Study with an 8-Year Follow-Up. Gerontology. 2012;58(4):296-304.

25. Jacobson BH, Smith D, Fronterhouse J, Kline C, Boolani A. Assessment of the Benefit of Powered Exercises for Muscular Endurance and Functional Capacity in Elderly Participants. J Phys Act Health. 2012;9(7):1030-5. 